\title{
Quantitative fluorimetrische Bestimmung der 4-Aminobuttersäure aus Liquor mit einem Aminosäurenanalysator
}

\author{
Von S. Uhlhaas und K. Olek \\ Institut für Humangenetik der Universität Bonn
}

(Eingegangen am 21. Dezember 1983/17. Februar 1984)

Zusammenfassung: Wir beschreiben eine schnelle, empfindliche und quantitative Bestimmungsmethode der 4-Aminobuttersäure aus menschlichem Liquor unter Verwendung eines modifizierten, handelsüblichen Aminosäurenanalysators mit Fluoreszenzdetektion.

Insbesondere wurden die hydrolytischen Eigenschaften der 5-Sulfosalicylsäure, die wesentlichen Einfluß auf das Meßergebnis haben, im Liquor untersucht.

Aus der gleichen Probenvorbereitungsmethode mit verschiedenen Mengen an Sulfosalicylsäure (50 g/l und $5 \mathrm{~g} / \mathrm{l})$ resultierten unterschiedliche Ergebnisse für 4-Aminobuttersäure (231,6 \pm 46,6 und $90.0 \pm 24,9$ $\mathrm{nmol} / 1)$.

Eine Enteiweißung des Liquors durch Ultrafiltration ergab sehr viel niedrigere Meßwerte $(57,6 \pm 16,0$ $\mathrm{nmol} / \mathrm{l})$. Durch eine Minimierung der Zeit zwischen Lumbalpunktion und Messen der Probe sowie Einsatz von Ultrafiltern konnten wir nachweisen, daß die nach Sulfosalicylsäureenteiweißung ( $5 \mathrm{~g} / \mathrm{l}$ Liquor) gemessenen Kontrollwerte für 4-Aminobuttersäure nur unwesentlich über den wahren Werten liegen.

Quantitative fluorometric measurement of 4-aminobutyric acid in human cerebrospinal fluid with an amino acid analyser

Summary: A rapid and simple procedure is described for the determination of 4-aminobutyric acid in human CSF. The $o$-phthaldialdehyde derivative of 4-aminobutyric acid is analysed with a slightly modified commercially available Amino Acid Analyser.

We investigated the sample preparation with special emphasis on the sulphosalicylic acid-induced hydrolysis of 4-aminobutyric acid containing compounds in human liquor. Our experiments demonstrate that the amount of estimated 4-aminobutyric acid depends considerably on the sulphosalicylic acid concentration used for protein precipitation.

Application of ultrafiltration instead of sulphosalicylic acid precipitation resulted in markedly decreased 4aminobutyric acid values. By first using ultrafiltration and by minimizing the time between lumbar puncture and analysis, it was shown that protein precipitation with a concentration of sulphosalicylic acid as low as $5 \mathrm{~g} / \mathrm{l}$ gives 4-aminobutyric acid values in CSF that are essentially correct.

\section{Einfüihrung}

4-Aminobuttersäure (GABA) wird als einer der wichtigsten inhibitorischen Neurotransmitter im ZNS betrachtet. Eine Bedeutung der 4-Aminobuttersäure als diagnostisches Kriterium bei verschiede- nen neurologischen Störungen ist somit wahrscheinlich $(1,2,3)$.

Die Bestimmung der 4-Aminobuttersäure-Konzentration im Gehirn durch direkte Gewebsentnahme ist zwar durchführbar, wegen des damit verbunde- 
nen Aufwandes und der Belastung des Patienten jedoch nicht sinnvoll.

$\mathrm{Da}$ die 4-Aminobuttersäure-Konzentration des $\mathrm{Li}$ quors im nanomolaren Bereich vorliegt, war es Ziel einiger Arbeiten nach 1976, genügend empfindliche und spezifische Methoden der 4-AminobuttersäureBestimmung aus Liquor zu entwickeln $(4,5)$.

Weithin angewendet wird die sogen. Radiorezeptormethode. Das Prinzip dieser Methode ist die Verdrängung von $\left[{ }^{3} \mathrm{H}\right] 4$-Aminobuttersäure, die an spezifische, synaptische Rezeptoren im Rattengehirngewebe gebunden ist, durch die im Liquor befindliche 4-Aminobuttersäure $(6,7,8)$. Nachteilig ist die verhältnismäßig geringe Spezifität. Beispielsweise besitzt das in wenigstens 10 facher Konzentration im Liquor vorkommende Homocarnosin (His-GABA) ebenfalls meßbare Affinität zu den 4-Aminobuttersäure-Rezeptoren (9). Die aufgrund dieser Methode ermittelten 4-Aminobuttersäure-Konzentrationen variierten von 230 bis über $500 \mathrm{nmol} / \mathrm{l}$.

Eine andere Methode ist die Verwendung eines gekoppelten Gaschromatographie-Massenspektrometrie-Systems (GC-MS System) $(10,11)$. Die komplizierte Technik dieser Analysensysteme erfordert hochqualifiziertes Personal und einen außerordentlich hohen finanziellen Aufwand. Kaum ein klinisch chemisches Labor verfügt über derartige Voraussetzungen. Ein zusätzlicher Nachteil ist die Hydrolyse konjugierter Formen der 4-Aminobuttersäure während der Derivatisierungsreaktion vor der GC-MS Analyse. Die Meßergebnisse von 100-600 nmol/ sind mit denen der Radiorezeptormethode vergleichbar.

Eine dritte, weit verbreitete Methode ist die flüssigkeitschromatographische Trennung der 4-Aminobuttersäure an einer Kationenaustauschersäule. Die eluierte 4-Aminobuttersäure wird mit $o$-Phthaldialdehyd in ein fluoreszierendes Derivat überführt und mit einem Fluoreszenzdetektor vermessen.

Bis 1980 wurden mit dieser Methode durchschnittliche 4-Aminobuttersäure-Konzentrationen im Liquor bei neurologisch normalen Kontrollgruppen zwischen 220 und $239 \mathrm{nmol} / \mathrm{l}$ gemessen $(4,5)$.

Mit einer modifizierten Ionenaustauscher-Fluoreszenzmethode stellten Perry et al. 1982 fest, daß mit $87 \mathrm{nmol} / \mathrm{l}$ die Liquorwerte für 4-Aminobuttersäure bei Normalpersonen wesentlich niedriger liegen (12).

Hier wurde besondere Aufmerksamkeit darauf verwendet, die Entstehung neuer 4-Aminobuttersäure aus ihren konjugierten Verbindungen zu minimie- ren. Dies geschah durch eine 10 fach verrringerte Menge an Sulfosalicylsäure $(5 \mathrm{~g} / \mathrm{l})$ zur Proteinfällung. Zudem wurde durch ein langes Analysenprogramm (260 min) die Trennung der 4-Aminobuttersäure von ihren Nachbarpeaks erheblich verbessert. Selbst bei Verwendung einer geringen Menge Sulfosaliclysäure zur Enteiweißung ist der Einfluß auf das Meßergebnis aber nicht auszuschließen.

Um dies quantitativ zu erfassen, ersetzten wir die Proteinfällung durch Ultrafiltration. Zum anderen bemühten wir uns, bei gleichbleibender Trennqualität den technischen und zeitlichen Aufwand der Bestimmung gegenüber der Prozedur von Perry et al. 1982 zu verringern (12).

\section{Methoden und Material}

Reagenzien

Zur Herstellung des Elutionspuffers und der Derivatisierungslösung wurden folgende Chemikalien der Firma E. Merck, Darmstadt, verwendet:

tri-Lithiumcitrat-4-hydrat zur Aminosäurenanalyse

Salzsäure $32 \%$ ig p.A.

Lithiumhydroxid-1-hydrat zur Aminosäurenanalyse

Brij 35

Borsäure krist. p.A.

o-Phthaldialdehyd für die Fluoreszenzanalyse

2-Mercaptoethanol zur Synthese

Zur Probenvorbereitung wurde 5-Sulfosalicylsäure-2-hydrat p. A. der Firma E. Merck, Darmstadt, yerwendet.

Als Standard diente der Amino Acid Calibration Standard Type PAN-B der Firma Benson, Reno, NV und 4-Aminobuttersäure p.A. der Firma Serva, Heidelberg.

Zur Herstellung aller wäßrigen Lösurigen verwendeten wir Wasser für die Hochleistungsflüssigkeitschromatographie der Firma J. T. Baker Chemicals B.V. Deventer NL.

\section{4-Aminobuttersäure-Analyzer}

Das von uns verwendete flüssigkeitschromatographische System ist aus in der Bundesrepublik handelsüblicheñ Elementen zusammengestellt worden.

Zur Förderung des Eluenten und der Derivatisierungslösung wählten wir je eine Dosapro Kolbenpumpe Model 19633 der Firma Milton Roy, Frankreich. Die thermostatisierbare Trennsäule hatte eine Länge von $250 \mathrm{~mm}$, einen inneren Durchmesser von $6 \mathrm{~mm}$ und war mit dem Kationenaustauscher Durrum DC 4A bis zu einer Höhe von $200 \mathrm{~mm}$ gefüllt. (Biotronik $\mathrm{GmbH}$, Müncheñ).

Das gesamte Fluß-System einschließlich des Reaktionscoils zur Ausbildung der 0 -Phthaldialdehyd-Derivate und der Schleifen zur Pulsationsdämpfung der Förderpumpen béstand aus Teflonschlauch mit einem inneren Durchmesser von $0,3 \mathrm{~mm}$ (Biotronik $\mathrm{GmbH}$, München).

Zur Fluoreszenzmessung der 0 -Phthaldialdehyd-Reaktionsprodukte verwendeten wir ein Filterfluorimeter Model FL-1A der Firma Gilson Medical Electronics, Middleton, WI, mit einer Anregungswellenlänge von $340 \mathrm{~nm}$ und' einer Emissionswellenlänge 
von $470 \mathrm{~nm}$. Die Detektorsignale wurden auf einem potentiometrischen Schreiber dargestellt (PM 8000, Phillips, NL).

Um ein genügend großes Signal für 4-Aminobuttersäure im Fluorimeter messen zu können, injizierten wir jeweils $500 \mu \mathrm{l}$ proteinfreien Liquors auf die Trennsäule. Die Elution der Aminosäuren erfolgte durch cinen einzigen $0,067 \mathrm{~mol} /$ Lithiumcitratpuffer mit einem pH-Wert von 4,60. Zur Entfernung des Ammoniaks und sonstiger leicht flüchtiger Verunreinigungen erhitzten wir den Elutionspuffer auf $110^{\circ} \mathrm{C}$ unter Rückfluß und anschließender Filtration über einen $0.22 \mu \mathrm{m}$ Nitrocellulosefilter der Firma Millipore, Bedford, Massachusetts.

In Abstimmung mit der Eluentenzusammensetzung und der Säulentemperatur von $65^{\circ} \mathrm{C}$ ergab eine Flußrate des Eluenten von 41 $\mathrm{ml} / \mathrm{h}$ die besten Trennergebnisse.

Dic Vorteile des sogen. single buffer system zeigen sich in einer von Puffersprüngen freien Basislinie (s. Abb. 1). Durch eine Optimicrung des Verhältnisses $\mathrm{pH}$-Wert/Ionenkonzentration konnten wir auf eine Vorwaschsäule (Durrum DC3 Kationenaustauscher) zur Verzögerung des Ammoniakdurchbruches verzichten. Diese Maßnahme war notwendig, weil das Vorwaschsäulenharz bei den erforderlichen hohen Empfindlichkeitsstufen nicht genügend hohe Retentionsfähigkeiten für 0 -Phthaldialdehyd-positive Substanzen bewies, was in Basislinienstörungen im Bereich der 4-Aminobuttersäure resultierte.

Zum Ansatz der Derivatisierungslösung stellten wir $5.00 \mathrm{ml}$ Borsäure $(61,8 \mathrm{~g} / \mathrm{l})$ mit Lithiumhydroxid $(25.2 \mathrm{~g} / \mathrm{l})$ auf $\mathrm{pH} 10,60$ ein. Dem Boratpuffer fügten wir $4 \mathrm{ml}$ 2-Mercaptoethanol, $10 \mathrm{ml}$ einer ethanolischen 0 -Phthaldialdehydlösung $(40 \mathrm{~g} / \mathrm{l})$ sowie $2 \mathrm{ml}$ Brij 35-Lösung $(350 \mathrm{~g} / \mathrm{l})$ hinzu. Die maximale Empfindlichkeit der Derivatisierungsreaktion lag im pH-Bereich von 9,3 bis 9,5. Daraus leiteten sich Föderraten für die Reagenzlösung von $55 \mathrm{ml} / \mathrm{h}$ ab.

Den Nachweis, daß die 4-Aminobuttersäure tatsächlich von den anderen physiologischen Aminosäuren getrennt wurde, führten wir mittels eines Kalibrationsstandards mit 35 Aminosäuren (Benson Type PAN-B, Reno, NV) und der reinen 4-Aminobuttersäure-Testsubstanz (Serva, Heidelberg).

Die Retentionszeit des 4-Aminobuttersäure-Peaks betrug $60 \mathrm{~min}$. Die Nachweisgrenze betrug 1-2 pmol, was dem 3-fachen Wert des Grundrauschens entspricht.

\section{Probensammlung und -vorbereitung}

Alle Kontrollpersonen unterzogen sich vormittags zwischen 8 und 11 Uhr in der Neurochirurgischen Klinik der Universität Bonn einer Lumbalpunktion. Punktiert wurde zwischen dem 3. und 4. Lendenwirbel zwecks Injektion eines Kontrastmittels für ein Myelogramm. Die ersten $2 \mathrm{ml}$ Liquor wurden in ein gekühltes Probenröhrchen getropft.

Die Liquores der 1 . Kontrollgruppe $(n=21)$ wurden sofort nach der Punktion mit krist. Sulfosalicylsạure ( $50 \mathrm{~g} / 1$ Liquor) versetzt. Nach Zentrifugation der Proteinfällungẹn wurdẹn die Úberstände bis zur Analyse bei $-40^{\circ} \mathrm{C}$ gelagert.

Die Punktate der 2. Kontrollgruppe $(n=17)$ wurden mit $5 \mathrm{~g}$ krist. Sulfosalicylsäure ( $5 \mathrm{~g} / \mathrm{L}$ Liquor) enteiweißt und die Uberstände bei $-40^{\circ} \mathrm{C}$ gelagert. Der Sulfosalicylsäuregehalt dieser Proben entspricht dem von Perry et al. 1982 beschriebenen (12).

Keine der so vorbereiteten Proben lagerte länger als 3 Tage.

Die Liquores der 3. Kontrollgruppe $(n=10)$ wurden in 2 Teile geteilt. Ein Teil wurde unter Verwendung eines Ultrafilters mit einer Ausschlußgrenze von 10000 Dalton ultrafiltriert (Immersible CX 10, Millipore, Bedford, Massachusetts), der andere Teil mit krist. Sulfosalicylsäure $(5 \mathrm{~g} / \mathrm{Liquor})$ enteiweißt.

Das so gewonnene Probenmaterial wurde sofort analysiert und die beiden Meßwerte miteinander verglichen.

\section{Ergebnisse}

Abbildung 1 zeigt typische Chromatogramme einer Standardlösung mit 35 Aminosäuren (a) mit 90 pmol/Injektion für jede Aminosäure und einer mit Sulfosalicylsäure enteiweißten Liquorprobe mit 80 nmol/1 4-Aminobuttersäure (b).

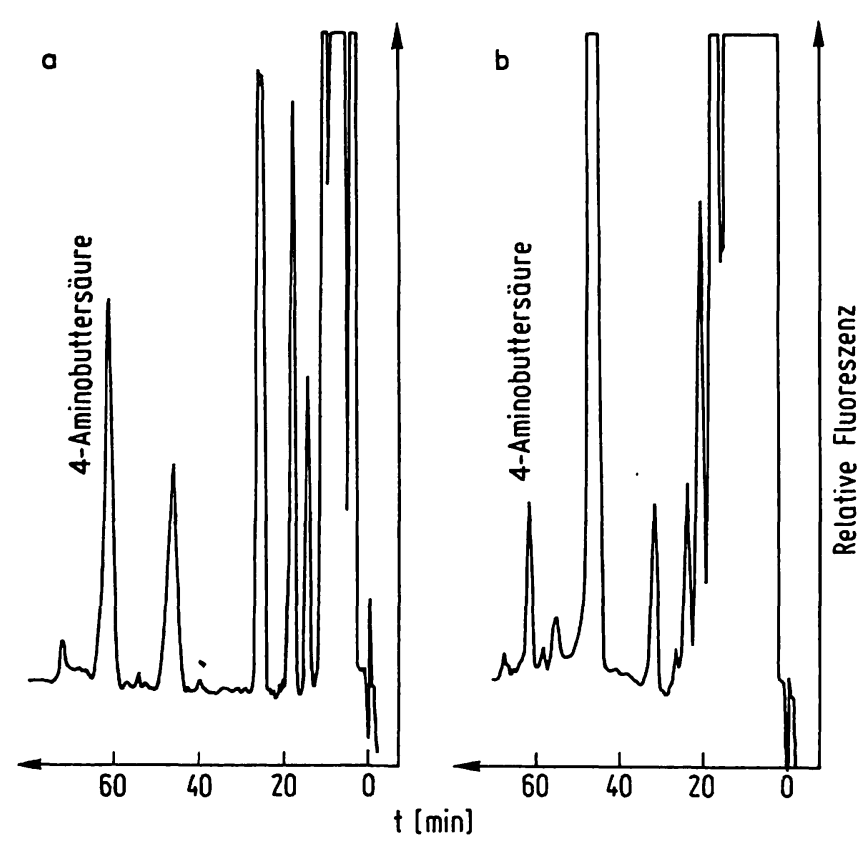

Abb. 1. a) Chromatogramm einer Standardlösung mit 35 Aminosäuren ( $90 \mathrm{pmol} /$ Aminosäure).

b) Chromatogramm von $500 \mu \mathrm{l}$ menschlichen Liquors, der mit Sulfosalicylsäure ( $5 \mathrm{~g} / \mathrm{l}$ Liquor) enteiweißt wurde (4-Aminobuttcrsäure: $80 \mathrm{nmol} / \mathrm{l}$ ).

Die in Abbildung 2 gezeigte Standardgerade erstellten wir durch Injektionen verschiedener Mengen an 4-Aminobuttersäure-Standard. Sie zeigt die gute $\mathrm{Li}$ nearität des Fluorimeters und der Derivatisierungsreaktion zwischen 3 und $150 \mathrm{pmol} /$ Injektion.

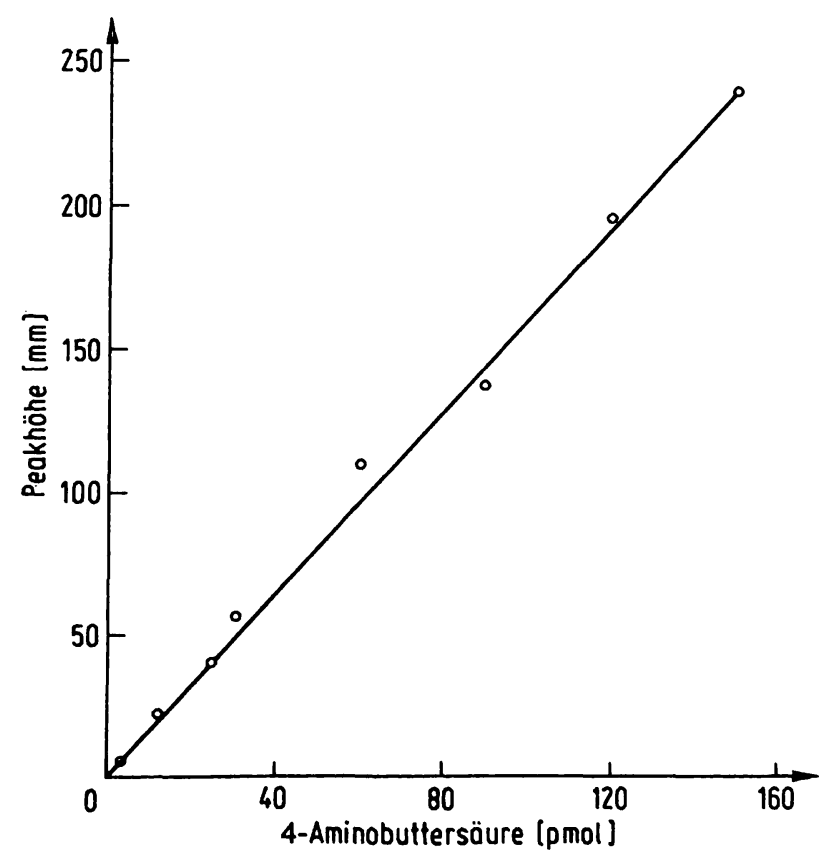

Abb. 2. Standardgerade für die 4-Aminobuttersüure-Bestimmung zwischen 3 und 150 pmol 4-Aminobuttersäure pro Injektion. 
Wiederholungsmessungen mit 50 pmol 4-Aminobuttersäure ergaben Standardabweichungen von $\pm 2,96$ ( $n=12$ ).

Die Wiederfindungsrate der Methode ermittelten wir durch Zugabe von bekannten Mengen 4-Aminobuttersäure-Standard zu Liquores. Sie betrug im Mittel $98,6 \%$ bei einer Standardabweichung von $\pm 2,95$ ( $\mathrm{n}=10$.

Trennungen von Testgemischen mit 35 Aminosäuren und Vergleiche mit Chromatogrammen einer bekannten Menge 4-Aminobuttersäure-Standard zeigten keine Koelution der 4-Aminobuttersäure mit anderen Aminosäuren.

Die Ergebnisse von 4-Aminobuttersäure-Bestimmungen der 1 . Kontrollgruppe $(n=21)$ lagen im Mittel bei $231,6 \mathrm{nmol} / 1 \mathrm{mit}$ einer Standardabweichung von $\pm 46,6$ und einem Bereich von 168,6 bis $316,8 \mathrm{nmol} / 1$ bei einer Sulfosalicylsäurekonzentration von $50 \mathrm{~g} / 1$ Liquor.

Böhlen et al. (4) sowie Hare \& Manyam (5), die zur Enteiweißung eine Sulfosalicylsäurekonzentration von $200 \mathrm{~g} / \mathrm{L}$ Liquor verwendeten, kamen mit $220 \pm$ 81 bzw. $239 \pm 76 \mathrm{nmol} / \mathrm{l}$ zu vergleichbaren Werten $(4,5)$.

Die Ergebnisse von 4-Aminobuttersäure-Bestimmungen der 2. Kontrollgruppe $(n=17)$ lagen im Mittel bei $90,0 \mathrm{nmol} / 1$ mit einer Standardabweichung von $\pm 24,9$ und einem Bereich von 28,1 bis $133,0 \mathrm{nmol} / 1$.

Der Anteil der Sulfosalicylsäure zur Proteinfällung betrug hier mit $5 \mathrm{~g} / \mathrm{l}$ Liquor nur $1 / 10$ der Menge der 1. Kontrollgruppe.

Die Meßwerte lagen signifikant niedriger als die in der 1. Kontrollgruppe und sind mit denen von Perry et al. zu vergleichen (12).

Die Ergebnisse von 4-Aminobuttersäure-Bestimmungen aus ultrafiltrierten Liquores der 3. Kontrollgruppe $(\mathrm{n}=10)$ lagen im Mittel bei $57,6 \mathrm{nmol} / 1 \mathrm{mit}$ einer Standardabweichung von $\pm 16,0$ und einem Bereich von 31,1 bis $73,3 \mathrm{nmol} / \mathrm{l}$.

Bei der Ultrafiltration wurden Verluste der Aminosäuren beobachtet. Deshalb wurden die Ausbeuten an 4-Aminobuttersäure mit 10 Ultrafiltern bestimmt. Sie betrugen im Mittel 50,1\% bei einem Bereich von 42,1 bis $55,7 \%$.

Das Ergebnis des ultrafiltrierten Teils wurde mit dem 4-Aminobuttersäure-Meßwert des mit $5 \mathrm{~g} / \mathrm{l}$ Sulfosalicylsäure enteiweißten Teils verglichen (s. Tab. 1).
Tab. 1. 4-Aminobuttersaure-Konzentrationen im menschlichen Liquor bei unterschiedlichen Probenvorbereitungsmethoden.

\begin{tabular}{|c|c|c|c|}
\hline \multirow{2}{*}{$\begin{array}{l}\text { 1. Kontroll- } \\
\text { gruppe } \\
\text { Sulfosalicyl- } \\
\text { säure } \\
\text { (50 g/l Liquor }\end{array}$} & \multirow{2}{*}{$\begin{array}{l}\text { 2. Kontroll- } \\
\text { gruppe } \\
\text { Sulfosalicyl- } \\
\text { säure } \\
\text { ( } 5 \text { g/l Liquor) }\end{array}$} & \multicolumn{2}{|c|}{ 3. Kontrollgruppe } \\
\hline & & Uiltrafilter & $\begin{array}{l}\text { Sulfosalicyl- } \\
\text { säure } \\
\text { ( } 5 \text { g/l Liquor) }\end{array}$ \\
\hline $\begin{array}{l}\text { Lagerung } \\
\text { bei }-40^{\circ} \mathrm{C}\end{array}$ & $\begin{array}{l}\text { Lagerung } \\
\text { bei }-40^{\circ} \mathrm{C}\end{array}$ & $\begin{array}{l}\text { Direkte } \\
\text { Analyse }\end{array}$ & $\begin{array}{l}\text { Direkte } \\
\text { Analyse }\end{array}$ \\
\hline \multicolumn{4}{|c|}{ 4-Aminobuttersäure (nmol/l) } \\
\hline $\begin{array}{l}283,9 \\
271,9 \\
223,0 \\
316,8 \\
301,3 \\
281,4 \\
306,8 \\
256,1 \\
214,4 \\
200,4 \\
182,5 \\
224,6 \\
190,1 \\
245,7 \\
225,9 \\
217,3 \\
198,6 \\
168,1 \\
183,1 \\
170,9 \\
199,8\end{array}$ & $\begin{array}{r}107,9 \\
84,1 \\
111,5 \\
89,4 \\
104,6 \\
61,3 \\
97,9 \\
133,0 \\
76,5 \\
99,6 \\
98,1 \\
99,7 \\
105,7 \\
50,1 \\
96,3 \\
28,1 \\
86,7\end{array}$ & $\begin{array}{l}73,3 \\
60,1 \\
71,1 \\
31,1 \\
72,1 \\
66,9 \\
51,9 \\
70,5 \\
42,9 \\
36,3\end{array}$ & $\begin{array}{l}86,9 \\
75,3 \\
81,1 \\
93,7 \\
88,9 \\
79,5 \\
58,6 \\
67,9 \\
44,7 \\
54,7\end{array}$ \\
\hline $\begin{array}{r}231,6 \\
\pm 46,6\end{array}$ & $\begin{array}{r}90,0 \\
\pm 24,9\end{array}$ & $\begin{array}{r}57,6 \\
\pm 16,0\end{array}$ & $\begin{array}{r}73,1 \\
\pm 16,2\end{array}$ \\
\hline
\end{tabular}

Es zeigte sich, daß die auf $100 \%$ Ausbeute umgerechneten 4-Aminobuttersäure-Werte in ultrafiltrierten Proben im Mittel um 15,5 nmol/1 niedriger lagen.

\section{Diskussion}

Die von uns ermittelten 4-Aminobuttersäure-Konzentrationen im Liquor von Kontrollpersonen sind wesentlich niedriger, als die von anderen Arbeitsgruppen, welche die Radiorezeptormethode. $(6,7)$, die GC-MS Methode $(10,11)$ oder die LC/HPLC Methode mit Fluoreszenżdetektion $(4,5)$ anwendeten.

Gebundene 4-Aminobuttersäure, z.B. in Homocarnosin (His-GABA), das in wenigstens 10facher Menge der 4-Aminobuttersäure im Liquor enthalten ist oder 4-Aminobutyrylcholin wirken sich bei allen Methoden nachteilig auf das Meßergebnis aus. Es ist bekannt, daß Homocarnosin $2 \%$ der Affinität der 4Aminobuttersäure gegenüber GABA-Rezeptoren besitzt (9). 
Konjugierte 4-Aminobuttersäure-Verbindungen sind bei der Derivatisierung vor der GC-MS Analyse einer zumindest teilweisen Zersetzungsreaktion unterworfen. Das Ansäuern des Liquors mit Sulfosalicylsäure bei der Proteinfällung vor der LC/HPLC Analyse bewirkt eine sogen. "On Column Hydrolysis" (12). Bei der Probenaufgabe wird durch die sauren chromatographischen Bedingungen an der Spitze der Säule neue 4-Aminobuttersäure aus Konjugaten freigesetzt, bis die Sulfosalicylsäure durch den Eluenten ausgewaschen ist. Die hohe Säulentemperatur von $65^{\circ} \mathrm{C}$ begünstigt diesen Vorgang.

Zusammenfassend kann gesagt werden, daß ähnliche Eigenschaften der 4-Aminobuttersäure-Konjugate gegenüber GABA-Rezeptoren (Radiorezeptorassay) bzw. die Bildung neuer 4-Aminobuttersäure durch Hydrolyse (GC-MS und LC/HPLC) zu überhöhten 4-Aminobuttersäure-Meßwerten führen.

Mit den Ergebnissen unserer Arbeit können wir die von Perry et al. 1982 erstmalig in solch niedrigen Konzentrationen bestimmten 4-AminobuttersäureLiquorkontrollwerte bestätigen (12). Vergleichsmessungen von Liquores, die mit Sulfosalicylsäure $(50 \mathrm{~g} / \mathrm{l}$ Liquor und $5 \mathrm{~g} / 1$ Liquor) enteiweißt worden waren (1. und 2. Kontrollgruppe), zeigten, daß mit der höheren Konzentration an Sulfosalicylsäure unter sonst gleichen methodischen Bedingungen mit 231,6 nmol/1 Liquor Meßergebnisse erzielt wurden, die mit denen früherer Arbeiten vergleichbar sind $(4,5)$.

Hier stellte sich uns die Frage, ob die mit einer Sulfosalicylsäurekonzentration von $5 \mathrm{~g} / 1$ Liquor behandelten Liquorproben nicht immer noch überhöhte 4Aminobuttersäure-Meßwerte ergeben. Wir versuchten dieses Problem zu lösen, indem wir die Sulfosalicylsäureproteinfällung durch Ultrafiltration ersetz= ten. Da die ultrafiltrierten Proben $\mathrm{pH}$-Werte zwischen 7 und 8 hatten, war eine saure Hydrolyse der 4-Aminobuttersäure-Konjugate auszuschließen. Die von Perry et al. 1982 beschrièbene On Column Hydrolysis dürfte bei einer ultrafiltrierten sulfosalicylšäurefreien Probe nur noch durch den Säuregehalt des Eluenten selbst hervorgerufen werden (12). Enzymatische Bildung neuer 4-Aminobuttersäure im Filtrat ist ebenfalls nicht wahrscheinlich, da alle Enzyme vom Ultrafilter zurückgehhalten werden sollten. Eine solch einfache und schnelle Gewinnung proteinfreien Liquors, bei dem keinerlei Nachfällungen mehr zu beobachten sind, wäre zweifellos vorteilhaft. Messungen von Teilen des gleichen ultrafiltrierten Liquors nach 1-10 Tagen Lagerung bei $-40^{\circ} \mathrm{C}$ zeigten ausgezeichnete Stabilität.
Die von uns beobachteten $o$-Phthaldialdehyd-positiven Auswaschungen der Millipore-Ultrafilter im $\mathrm{Be}-$ reich der sauren Aminosäuren war bei unserer Zielsetzung nicht relevant für das Analysenergebnis. Allerdings war der Untergrund im Bereich des Ammoniak und somit der 4-Aminobuttersäure bei ultrafiltrierten Proben größer als bei Sulfosalicylsäurebehandelten. Bei der Ultrafiltration von Aminosäurentestgemischen beobachteten wir Verluste bei den einzelnen Aminosäuren. Der Mittelwert der Ausbeuten für die 4-Aminobuttersäure betrug 50,1\% (n $=10$ ). Der relativ breite Bereich von $42,1-55,7 \%$ Ausbeute zeigt wenig spezifische Eigenschaften des Filtermaterials.

Durch die relativ niedrige Wiederfindungsrate ergeben sich allerdings Schreibersignale, die nahe bei der Nachweisgrenze liegen. Dies wirkt sich besonders dann aus, wenn Reagenz und Eluent mehrere Tage alt sind. Die Ergebnisse unserer Untersuchungen zeigen außerdem, daß nach einmaligem Einfrieren eines mit Sulfosalicylsäure ( $5 \mathrm{~g} / 1$ Liquor) enteiweißten Liquors etwa um $15 \mathrm{nmol} / \mathrm{l} \mathrm{mehr}$ 4-Aminobuttersäure gefunden wurde als in sofort analysierten Proben. Auf $100 \%$ Ausbeute umgerechnete 4-Aminobuttersäure-Werte ultrafiltrierter Liquores weisen eine um etwa $15 \mathrm{nmol} / 1$ geringere 4-Aminobuttersäure-Konzentration auf als die mit Sulfosalicylsäure ( $5 \mathrm{~g} / 1$ Liquor) enteiweißten Proben (s. Tab. 1).

Die Vorzüge unserer Methode gegenüber der von Perry et al. 1982 sind wesentlich kürzere Analysenzeiten (12). Wir benötigen statt 260 min nur noch 60 min bis zur Elution der 4-Aminobuttersäure bei gleich guter Trennqualität. Weiterhin ist die Handhabung unseres Analysenprogrammes einfacher, weil wir nur einen statt zwei Elutionspuffer gebrauchen. Der Einsatz von zwei Puffern ist deshalb problematisch, weil bei hohen Empfindlichkeitsstufen erstens die Puffersprünge in der Basislinie sehr groß sind und zweitens Verunreinigungen aus dem ersten Puffer mit dem zweiten eluiert werden. Dies stellt siçh in störendem Untergrund dar.

Den Einsatz von Ultrafiltern zur Probenenteiweißung können wir wegen der wenig spezifischen Eigenschaften des Filtermaterials nur bedingt empfehlen. Zur Ermittlung der wahren 4-Aminobuttersäure-Konzentrationen waren sie uns jedoch eine wertvolle Hilfe. Die Differenz der 4-AminobuttersäureMeßwerte zwischen ultrafiltrierten und mit Sulfosalicylsäure ( $5 \mathrm{~g} / 1$ Liquor) enteiweißten Liquorproben ist unwesentlich. Bei untergrundfreien Chromatogrammen liegen die mit Sulfosalicylsäure $(5 \mathrm{~g} / \mathrm{Li}$ quor) vorbereiteten Proben genügend nahe am tatsächlichen Wert. 
Die wahren 4-Aminobuttersäure-Liquorwerte liegen somit im Mittel bei $57,6 \mathrm{nmol} / 1$ mit einem Bereich von 31,1 bis $73,3 \mathrm{nmol} / \mathrm{l}$. Nach unseren bisherigen Ergebnissen ist die beschriebene Methode problemlos auf die 4-Aminobuttersäure-Bestimmung im Serum zu übertragen. Die Werte scheinen in der gleichen Größenordnung zu liegen wie im Liquor.

\section{Literatur}

1. Perry, T. L.. Hansen, S. \& Kloster, M. (1973) N. Engl. J. Med. 288. 337-342.

2. Langer, D. H., LaVonne Brown, G., Bunney, W. E. \& van Kammen, D. P. (1975) N. Engl. J. Med. 293, 201.

3. Emson, P. C. (1975) Int. J. Biochem. 6, 689.

4. Böhlen, P., Schlechter, P. J., van Damme, W., Coquillat, G., Dosch, J. C. \& Koch-Weser, J. (1978) Clin. Chem. 24, 256260).

5. Hare. T. A. \& Manyam, N. V. B. (1980) Anal. Biochem. 101 , 349-355

6. Enna, S. J.. Wood, J. H. \& Snyder, S. H. (1977) J. Neurochem. $28,1121-1124$
Eine entsprechende Untersuchung wird zur Zeit von uns durchgeführt.

\section{Danksagung}

Wir bedanken uns bei Frau Priv.-Doz. Dr. M. Rao und Herrn Dipl.-Chem. A. H. Edelbi für die freundliche Hilfe beim Beschaffen der Liquor-Proben.
7. Baraczka, K. \& Sperk, G. (1981) Clin. Chim. Acta 109, 7782.

8. Abbott, R. J., Keidan, J., Pye, I. F. \& Nahorski, S. R. (1981) J. Neurochem. 37, 1042-1044.

9. Enna, S. J. \& Synder, S. H. (1976) J. Neurochem. 26, 221224.

10. Huizinga, J. D., Tellken, A. W., Muskiet, F. A. J., Jeuring, H. J. \& Wolthers, B. G. (1978) J. Neurochem. 30, 911-913.

11. Faull, K. F., DoAmaral, J. R., Berger, P. A. \& Barchas, J. D. (1978) J. Neurochem. 31, 1119-1122.

12. Perry, T. L., Hansen, S., Wall, R. A. \& Gauthier, S. G. (1982) J. Neurochem. 38. 766-773.

Siegfried Uhlhaas Institut für Humangenetik der Universität Bonn Wilhelmstraße 31 D-5300 Bonn 1 